DOI: 10.20472/IAC.2019.052.033

\author{
LOYISO LUVALO \\ University of South Africa, South Africa
}

\title{
UBUNTU PHILOSOPHY ON DEALING WITH PATRIACHY
}

\begin{abstract}
:
South Africa still shows signs of being a very patriarchal society, according to literature and this somehow leads to instances where rights of women and children are trampled on because of male-favouring environment. This seems to be the case despite the constitution and current laws that are prohibiting discrimination on grounds of one's gender. Applying Ubuntu philosophy, on how patriarchy can be a remedy in today's society as opposed to perpetuating ignorance of both women and children's rights. The paper draws on the Eastern Cape's chapter of South Africa's National Research Foundation (NRF) - funded 'Archaeology of Ubuntu's study to explore the notion of Ubuntu as a moral concept. Archaeology of Ubuntu study regards community elders, as the custodians of African epistemologies, who should be consulted while still alive because once they die all the wisdom and knowledge of cultures get lost with them. In this paper, it is argued that through Ubuntu philosophy, patriarchy in the olden days did not mean ignoring of rights of women and children. The paper finds that isiXhosa speaking elders through Ubuntu philosophy, patriarchy enabled caring of the vulnerable in the society, which are women, children and the elderly. Therefore, the conclusion is that Ubuntu philosophy can still be applied in remedying the ever-increasing incidents of trampling of women and children's right in a patriarchal South African society.
\end{abstract}

\section{Keywords:}

Ubuntu, patriachy, human rights, education and gender equality

JEL Classification: D63 